\title{
INVESTIGATION OF DIFFERENT MODELING APPROACHES FOR COMPUTATIONAL FLUID DYNAMICS SIMULATION OF HIGH-PRESSURE ROCKET COMBUSTORS
}

\author{
B. Ivancic ${ }^{1}$, H. Riedmann ${ }^{1}$, M. Frey ${ }^{1}$, O. Knab ${ }^{1}$, S. Karl ${ }^{2}$, \\ and K. Hannemann ${ }^{2}$ \\ ${ }^{1}$ Airbus Defence and Space \\ Munich 81663, Germany \\ ${ }^{2}$ Deutsches Zentrum für Luft- und Raumfahrt (DLR) \\ Institute of Aerodynamics and Flow Technology \\ Göttingen 37073, Germany
}

The paper summarizes technical results and first highlights of the cooperation between DLR and Airbus Defence and Space (DS) within the work package "CFD Modeling of Combustion Chamber Processes" conducted in the frame of the Propulsion 2020 Project. Within the addressed work package, DLR Göttingen and Airbus DS Ottobrunn have identified several test cases where adequate test data are available and which can be used for proper validation of the computational fluid dynamics (CFD) tools. In this paper, the first test case, the Penn State chamber (RCM1 ), is discussed. Presenting the simulation results from three different tools, it is shown that the test case can be computed properly with steady-state Reynolds-averaged Navier-Stokes (RANS) approaches. The achieved simulation results reproduce the measured wall heat flux as an important validation parameter very well but also reveal some inconsistencies in the test data which are addressed in this paper.

\section{INTRODUCTION}

The main target of the Propulsion 2020 cooperation between DLR and Airbus DS is the strengthening of the knowledge and competence in the area of rocket propulsion combustion devices, particularly with regard to test, modeling, and simulation capabilities. One of the work packages is focused on CFD modeling of rocket combustion chambers whose first results are shown here. This work package is executed in close cooperation between DLR Institute of Aerodynamics and Flow Technology and Airbus DS. The objective is to develop numerical 
tools and methods which are applicable to real rocket thrust chamber hardware design and optimization. Hence, the tool packages must be designed to simulate multiinjector combustors in a reasonable time frame encompassing the driving mechanisms as propellant disintegration, combustion, and heat transfer. Conclusions on methods and models drawn from single-element combustor simulations as addressed in this paper are valuable but still insufficient if not transferable to multiinjector simulations since only the latter can support engine layout and contribute to reduce development time and cost.

Airbus DS's combustion modeling capabilities for $\mathrm{H}_{2} / \mathrm{O}_{2}$ thrust chambers cover the complete range from the two-phase subcritical regime up to trans- and supercritical conditions (single phase). For this purpose, a commercial CFD tool (ANSYS CFX) is adapted and the implemented models are extended for the usage at the extreme boundary conditions of rocket combustors. The second approach taken at Airbus DS is working with in-house CFD tools for the simulation of flow, combustion, and heat transfer in rocket thrust chambers. One of these in-house tools, Rocflam-II, has been operational for several years now and is a standard tool for design and analysis of rocket thrust chambers at Airbus DS. However, as working with in-house tools needs continuous development, there is a dedicated successor for Rocflam-II, consequently called Rocflam3, which has been applied for the work discussed in this paper. Additional computations with the DLR TAU Code have been performed to supplement the CFX and Rocflam3 investigations with data from alternative combustion and turbulence modeling approaches. The primary intention of the application of diverse numerical tools in the present work is to provide a broad spectrum of data for the assessment of different modeling methodologies.

In order to achieve high efficiency and optimized mass relations, the thrust chambers used in modern rocket engines such as Vinci work at elevated pressure levels. At the same time, a very wide temperature range is covered inside the combustion chamber. The complexity of the dominant phenomena inside rocket combustors at these extreme thermodynamic conditions makes the modeling very difficult. Especially, modeling the mixing and combustion of the propellants determining the integral performance characteristics and the wall heat loading of propulsion systems is a very challenging task. The well-known single element Penn State test case $[1,2]$ provides test data for the validation of simulation tools in this field of application. In the present work, the three tools CFX, Rocflam3, and TAU are applied to this test case and the results are compared being aware that there are a variety of differences regarding the numerical and physical models implemented in each tool. Therefore, it is not the objective of this work to compare individual model approaches but rather to compare the complete set of models used in each tool. The present investigations show that the Penn State single element combustor test case can be simulated with RANS approaches leading to a very good agreement with the measured wall heat flux profile. Former publications (see, for example, $[3,4]$ ) have promoted the neces- 
sity of time-accurate simulations for this purpose as they have not been able to achieve good results with steady-state RANS methods. The results documented in this paper show that the referred statements give a wrong impression of what is possible with RANS simulations in the field of rocket thrust chamber simulations and what is not. This is important since up to now, only RANS methods have been demonstrated to be efficiently applicable to multiinjector combustor simulations.

\section{TEST CASE DESCRIPTION}

The Penn State combustion chamber configuration has been set up by NASA Marshall Space Flight Center and the Penn State University [1,2] in order to support the development of CFD tools for rocket combustor simulations with a test case for verification and validation. Therefore, wall heat flux and temperature measurements have been performed in a rocket combustor with a single coaxial injector operating on gaseous oxygen $\left(\mathrm{GO}_{2}\right)$ and hydrogen $\left(\mathrm{GH}_{2}\right)$. Both propellants enter the main chamber in a preburnt state which corresponds to a staged combustion cycle. The operating point lies at a chamber pressure of $p_{c}=5.42 \mathrm{MPa}$ and an overall mixture ratio of $\mathrm{O} / \mathrm{F} \approx 6.6$ which is a representative load point for rocket combustors. A schematic of this rocket chamber is shown in Fig. 1.

The main chamber has a circular cross section with a diameter of $d_{c}$ $=38.1 \mathrm{~mm}$ and a length of $l_{c}=285.75 \mathrm{~mm}$. The throat diameter is $d_{t}=8.2 \mathrm{~mm}$.

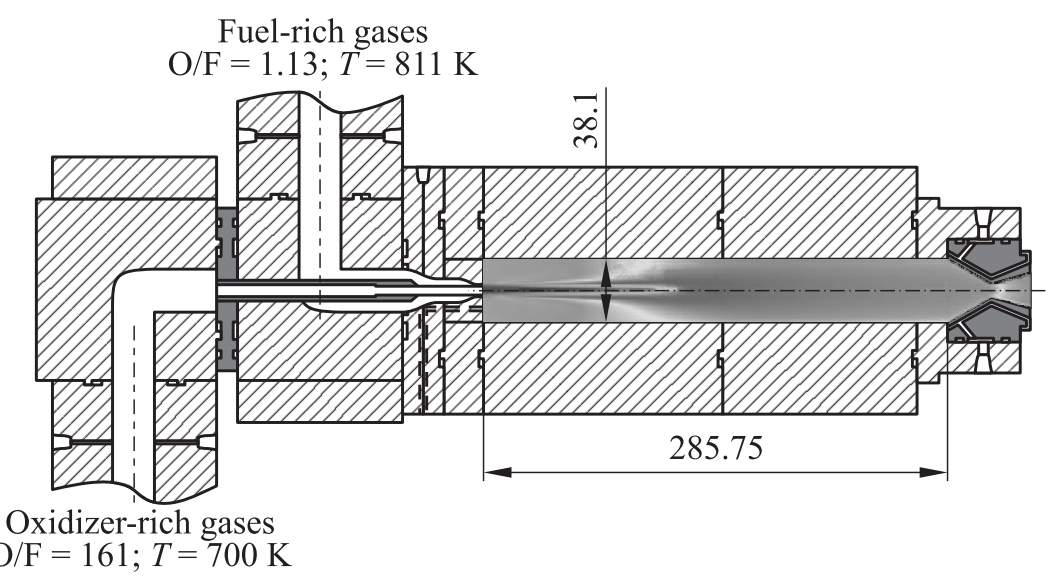

Figure 1 Schematic of the Penn State single element combustor with specified propellant inlet conditions [1]. Dimensions are in millimeters 
Table 1 Injector and main chamber quantities: measured values and calculated exhaust compositions of the preburners as specified in [1]

\begin{tabular}{lccc}
\hline \multicolumn{4}{c}{ Main chamber } \\
\hline Quantity & Unit & Value \\
\hline Chamber pressure & $\mathrm{MPa}$ & \multicolumn{3}{c}{5.42} \\
Total mass flow rate & $\mathrm{kg} / \mathrm{s}$ & \multicolumn{2}{c}{$123.5 \cdot 10^{-3}$} \\
Overall O/F & - & 6.6 \\
\hline \hline & \multicolumn{3}{c}{ Injector } \\
\hline Quantity & Unit & Oxidizer-rich mixture & Fuel-rich mixture \\
\hline Mass flow rate & $\mathrm{kg} / \mathrm{s}$ & $90.4 \cdot 10^{-3}$ & $33.1 \cdot 10^{-3}$ \\
$\mathrm{O} / \mathrm{F}$ & - & 161 & 1.13 \\
Temperature & $\mathrm{K}$ & 700 & 811 \\
$Y_{\mathrm{O}_{2}}$ & - & 0.945 & 0.0 \\
$Y_{\mathrm{H}_{2}}$ & - & 0.0 & 0.402 \\
$Y_{\mathrm{H}_{2} \mathrm{O}}$ & - & 0.055 & 0.598 \\
\hline
\end{tabular}

This results in a characteristic chamber length of $l^{*}=6.46 \mathrm{~m}$ which is clearly longer than in common $\mathrm{H}_{2} / \mathrm{O}_{2}$ rocket combustion chambers $\left(l^{*}<1 \mathrm{~m}\right)$. While the cylindrical part of the chamber is cooled capacitively, the throat section is cooled actively with cold water flowing through the cooling channels in the chamber wall. Wall temperature and heat flux measurements have been performed in the cylindrical part using coaxial thermocouples [1] with multiple measurement positions. While the wall temperature profile serves as thermal boundary condition for the CFD simulations, the main target is to compute the correct heat flux profile. Measured temperatures, mass flow rates, and mixture ratios are given for the two preburner flows and serve as inlet boundary conditions for the main chamber. The test case description also provides fuel and oxidizer preburner gas compositions. These quantities are not measured but computed under the assumption of chemical equilibrium. All these values can be found in [2] and are repeated in Table 1. Unfortunately, it has not been possible to reproduce the measured chamber pressure $p_{c}=5.42 \mathrm{MPa}$ with the given inflow conditions and the given chamber geometry neither in CEA2 [5] nor in the applied CFD tools indicating inconsistencies in the experimental data. This topic will be discussed in section 3 .

\section{INCONSISTENCY OF THE TEST DATA}

During the evaluation and analysis of the Penn State test case, Airbus DS exposed some inconsistencies in the measured test data provided, for example, in [1] where the measured combustion chamber pressure is given as $p_{c}=5.42 \mathrm{MPa}$. 
With the given measured propellant mass flow rate $\dot{m}$ and the throat area $A_{t}$, the characteristic velocity is determined as follows:

$$
c_{\mathrm{exp}}^{*}=\frac{p_{c} A_{t}}{\dot{m}}=2298.5 \mathrm{~m} / \mathrm{s} .
$$

The theoretical or ideal characteristic velocity can be calculated with the chemical equilibrium tool CEA2 [5]. The CEA2 calculation delivers an ideal characteristic velocity of $c_{\text {ideal }}^{*}=2041.4 \mathrm{~m} / \mathrm{s}$ for the inflow conditions given in Table 1 and additionally taking into account the heat losses along the chamber body. Dividing the experimental value by the ideal value results in a combustion efficiency of $\eta_{c^{*} \text {, Test }}=112.5 \%$. However, as values greater than $100 \%$ may not exist, something must be wrong. There must be some inaccuracies between specified and measured data. Apart from measurement errors, there are several possibilities to explain this gap. The most probable hypotheses are an incomplete preburner combustion or a geometric contraction of the throat area under thermal load. Furthermore, it is possible that inaccurate mass flow measurements contribute to this inconsistency.

A contraction of the throat with constant propellant mass flow rates directly results in a higher combustion chamber pressure. Assuming a realistic combustion efficiency of $99 \%$, a reduction of the throat diameter by $0.28 \mathrm{~mm}(3.4 \%)$ must have been given during the experiment. However, such a heavy distortion of the cooled nozzle is not realistic. This means the inconsistency cannot be completely explained only by this phenomenon.

An incomplete combustion in the two preburners can be a realistic explanation at least for the major part of this inconsistency. Both preburners run at very extreme mixture ratios meaning that the temperature of the burnt gases is rather low. Propellants which are not burnt in the stoichiometric flame sheet where the temperatures are very high may also not be burnt further downstream in the postflame region. This behavior is significantly different compared to the processes in the main combustion chamber where the temperatures also in the postflame region are relatively high and where chemical equilibrium is a very good assumption. In the preburners, the temperatures in the postflame region are very often below $900 \mathrm{~K}$, i. e., the chemical kinetics are very slow and chemical nonequilibrium actually can exist for $\mathrm{H}_{2} / \mathrm{O}_{2}$. This means that even for extreme mixture ratios, it is possible that both propellants survive unburnt in the preburner. In this case, the final combustion occurs in the main combustion chamber generating higher heat release and combustion pressure.

To solve a rocket problem in CEA2, the user specifies the chamber pressure, the inlet enthalpies, and the mixture ratio. The propellant mass flow rate is not a direct result from CEA2 but can be determined via the characteristic velocity computed by CEA2. In this case, the discussed inconsistency becomes visible as the computed propellant mass flow rate is much higher than the given one. However, in CFD simulations, at least when the nozzle part is included, the 
chamber pressure is a simulation result and not a boundary condition. This is because cold propellant mass flow rates are injected and the simulated efficiency of the combustion process determines the resulting chamber pressure. Therefore, the explained inconsistency becomes evident as the computed chamber pressure is far below the measured value. This will be addressed again in the discussion of the simulation results in section 5 .

\section{PRESENTATION OF THE APPLIED TOOLS, MODELS, AND SETTINGS}

In earlier times, the relevant processes inside rocket combustion chambers, especially the wall heat flux distribution, were predicted by engineering tools based on one-dimensional (1D) flow, chemical equilibrium, and Nusselt correlations. Further developments at Airbus DS led to the more sophisticated two-dimensional (2D) spray combustion tool Rocflam-II [6]. However, especially in multiinjector chambers, three-dimensional (3D) phenonema are existent and not negligible in certain parts of the combustion chamber. Therefore, high effort has been put in the in-house development of Rocflam3 (3D), which is the designated successor of Rocflam-II (2D), as well as in the adaptation of the commercial 3D-CFD solver CFX for the usage under these extreme thermodynamic conditions. Both Airbus DS CFD tools, i. e., CFX and Rocflam3, are presented in more detail (e.g., applied combustion models, mixing rules, etc.) together with the DLR TAU code in the following subsections.

\subsection{ANSYS CFX (Including Modifications Made by Airbus DS)}

Especially for the rocket applications of the commercial CFD tool CFX, the main problem is that this solver is developed for a broad range of applications (e.g., turbomachinery) and not focused on rocket propulsion. The adaption process of CFX and the first validation studies have been shown in [7]. Two different combustion models have been implemented and tested in CFX: the chemical equilibrium and the Flamelet approach. The equilibrium-based combustion model is not a CFX standard combustion model. It was developed and implemented via user-defined functions into CFX by Airbus DS. The Flamelet model is a more sophisticated model because it ensures accurate chemistry kinetics and accounts for nonequilibrium effects at low computational cost, because no species transport equations are solved but a mixture fraction equation. The influence of turbulence on combustion is treated by a presumed PDF (Beta- and clipped Gauss-pPDF) approach, where the main influencing factors for the PDF shape are the mixture fraction and its variance. The standard turbulence model 
applied in CFX for combustor simulation is the Menter shear stress transport (SST) model $[8,9]$.

The CFX simulation shown in this work applied the Flamelet combustion model and the SST model for turbulence [7,9] with a turbulent Lewis number unity approach $\left(\mathrm{Le}_{t}=1\right)$ and $\mathrm{Pr}_{t}=\mathrm{Sc}_{t}=0.85$.

For the simulations with CFX, the so-called dense gas model is implemented in trans- and supercritical conditions. The dense oxygen jet is completely described within the Euler equations and appropriate material property data bases are essential for accurate modeling of the mixing of the cryogenic and dense oxygen with the surrounding hot gases. The fluid properties in CFX are extended with real gas data and sophisticated mixing rules for viscosity and heat conductivity are implemented also via user-defined functions. For viscosity and thermal conductivity, the Brokaw and Wilke approaches are used [10]. By this approach, it is ensured that the mixing and, also, the subsequent combustion process at much higher temperatures are covered properly by the fluid data base. Moreover, validation studies revealed that the mixing laws are important in order to get accurate results. Especially for a correct simulation of the wall heat transfer, a proper modeling of the gas mixture properties is essential.

The computational domain for the Airbus DS CFD simulations performed with CFX and Rocflam3 starts at the beginning of the main chamber. The grid is shown in Fig. 2. The inner parts of the injector as well as the recess are not resolved. However, the post-tip between oxidizer and fuel inlet is resolved by the grid. Most authors who have published on this test case have started their simulations further upstream thus resolving the inner part of the injection element. This has also been done with CFX in an alternative approach. The

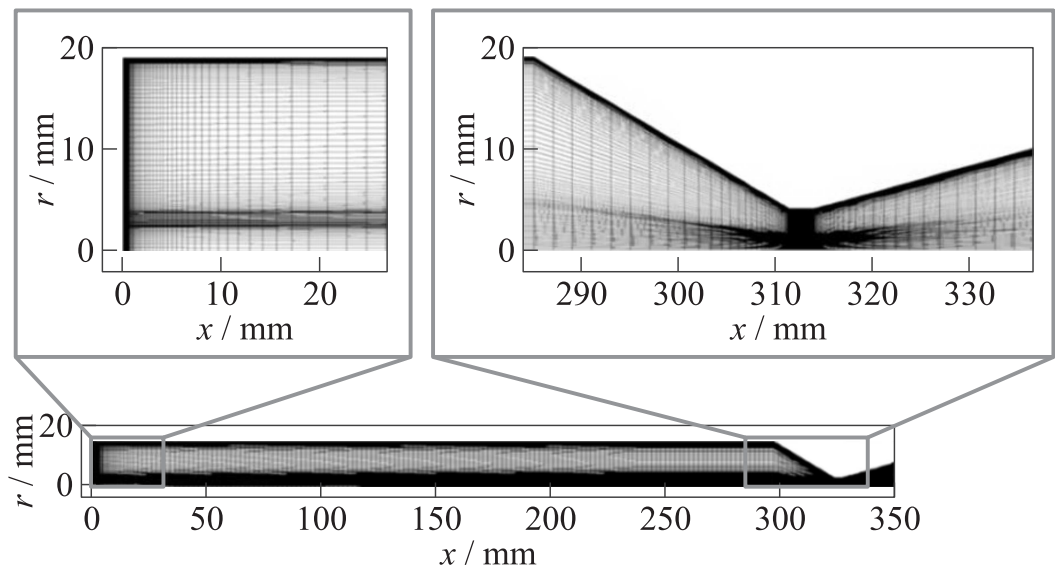

Figure 2 Computational grid for the CFX and Rocflam3 simulations 
results will be shown in subsection 6.2. As CFX is a $3 \mathrm{D}$ solver, the grid is a segment of one degree with one grid cell used for resolving the circumferential direction. This means that all simulations presented here are axisymmetric. The grid shown in Fig. 2 consists of 91,000 grid cells and is a result of an intensive grid study. A fine wall resolution ensures $y^{+}<1$ for the whole combustion chamber. Mass flows and temperatures are used as boundary conditions for the inlets of fuel and oxidizer, i. e., the combustor pressure is a simulation result. All walls are treated as smooth no-slip walls with prescribed temperatures as thermal boundary condition. The wall temperatures of the post-tip area between the fuel and oxidizer inlets as well as the faceplate are set to the constant value of $T_{w}=755 \mathrm{~K}$. This value is not measured in the experiment but seems realistic and has been used by many other authors (see, for example, [11]). The measured wall temperature profile is used as boundary condition for the chamber wall. Linear interpolation is applied to compute values between the measuring points. Downstream of the last measuring point, a constant wall temperature of $T_{w}$ $=511 \mathrm{~K}$ is prescribed. A supersonic outlet boundary condition is used in the outlet plane.

\subsection{Rocflam3}

The Airbus DS in-house tool Rocflam3 is a finite-volume solver for nonorthogonal, boundary-fitted and block-structured grids that applies the SIMPLE (semiimplicit method for pressure-linked equations) algorithm to solve the compressible RANS equations in three spatial dimensions. Rocflam3 features different $k-\varepsilon$ and $k-\omega$ turbulence models and an equilibrium-based pPDF-chemistry model which takes into account effects of turbulent combustion as well as real gas effects. The simulation of the Penn State test case shall contribute to the validation process of Rocflam3 which is still under development. For the present simulations, Rocflam3 uses an equilibrium-based pPDF-combustion model and the Launder-Sharma turbulence model [12] with $\operatorname{Pr}_{t}=0.9$ and $\mathrm{Sc}_{t}=0.6$ resulting in a turbulent Lewis number of $\mathrm{Le}_{t}=0.67$. Furthermore, the Durbin realizability constraint [13] and the Yap correction [14] are implemented in order to enhance modeling of the turbulent heat transfer.

Regarding the numerical mesh and the prescribed boundary conditions, the Rocflam3 simulations use values and settings which are identical to the ones used in the presented CFX simulations.

\subsection{DLR TAU Code}

The TAU code is a finite-volume flow solver for the Euler and Navier-Stokes equations including a wide range of turbulence modeling capabilities [15]. The 
present investigation is based on steady-state RANS computations using the Spalart-Allmaras [16] and Menter SST [8] eddy viscosity models. The AUSMDV flux vector splitting scheme was applied together with MUSCL (monotonic upwind scheme for conservation laws) gradient reconstruction to achieve secondorder spatial accuracy [17]. Combustion was modeled using a detailed-chemistry and a Flamelet approach.

The detailed-chemistry combustion model in the DLR Tau code is an extension of the models for chemical and thermal nonequilibrium flows in high enthalpy (re-)entry aerothermodynamics. The flow is considered to be a reacting mixture of thermally perfect gases. A transport equation is solved for each individual species. The chemical source term in this set of transport equations is computed from the law of mass action. The forward and backward reaction rates are computed using the modified Arrhenius law and equilibrium constants. Turbulence-chemistry interactions are modeled using an assumed probability density function (PDF) approach [18] which considers local fluctuation of temperature and species concentrations. Two sets of reaction mechanisms for hydrogen combustion were considered: a 19-step [18] and a reduced 7-step [19] mechanism based on the Jachimowski scheme. A detailed model description and performance assessment for steady and unsteady combustion problems is given in $[20,21]$.

The applied compressible Flamelet model for nonpremixed combustion [22] is a conserved scalar method. The flow field is assumed to consist of fuel and oxidizer at an arbitrary state of mixing which is characterized by a nonreactive scalar, the mixture fraction. Two additional transport equations for the mixture fraction and its variance are solved and the evaluation of the local chemical composition is performed on the basis of a precomputed lookup-table (Flamelet library). The flame properties and the species composition inside the flame zone are governed by the thermodynamic state and composition of the fuel and oxidizer stream and the scalar dissipation rate of the mixture fraction which is related to the variance of the mixture fraction and the specific turbulent dissipation rate. The Flamelet library was computed with the FlameMaster package [23] and was based on a 19-step hydrogen combustion mechanism described by Pitsch [24].

The thermodynamic properties (energy, entropy, and specific heat) are calculated using the partition functions for each individual species in the reacting gas mixture. Knowing the mixture composition and the thermodynamic state of the individual species, the properties of the reacting gas mixture are computed using suitable mixture rules [20]. The species diffusion fluxes are modeled using Fick's law applying an averaged diffusion coefficient for all species This approximate diffusion coefficient is computed using the laminar and eddy viscosities and constant Schmidt numbers of $\mathrm{Sc}=\mathrm{Sc}_{t}=0.6$. The turbulent heat conductivity was computed assuming a constant ratio of laminar to turbulent Prandtl numbers of 0.85 . 


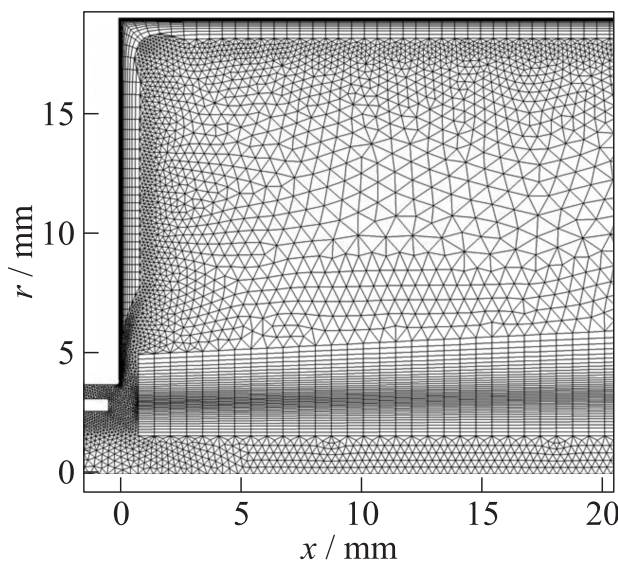

Figure 3 Computational grid (TAU, standard grid density) in the vicinity of the injector

The computational domain shown in Fig. 2 was also used for the $2 \mathrm{D}$ axisymmetric TAU simulations. A small section of the coaxial injector was added at the inflow boundary (visible in Fig. 3). The computational plane was meshed with a hybrid grid. The near-wall regions were discretized with structured quadrilateral sublayers. The wallnormal size of the wall-adjacent cell ranging between 1 and $0.5 \mu \mathrm{m}$ was chosen to ensure a nondimensional wall distance of $y^{+}<1$ for the first cell (as needed by the applied low-Reynolds turbulence models) and a sufficient resolution of the temperature gradients close to the wall. The inner flow field was discretized with an unstructured triangular grid with an embedded structured block for better resolution of the shear layer and flame zone. A detail of the applied grid (medium resolution) in the vicinity of the injector is shown in Fig. 3).

The combustor walls were treated using a noncatalytic, no-slip boundary condition with a prescribed temperature profile taken from the experimental test case description [2]. The inflow at the coaxial injector was modeled using a mass flow boundary condition with prescribed mass flow rates, species concentrations, and inflow temperatures according to Table 1.

\section{EVALUATION AND COMPARISON OF THE FINAL SIMULATION RESULTS}

This section shows the final simulation results obtained with CFX, Rocflam3, and TAU. Extensive parameter studies have been conducted with each tool in order to develop the final settings. In this process, three different model packages have been designed to perform the same task. The modeling approaches are not identical for the three tools. Therefore, as it can be seen in Fig. 4, there are, of course, differences between the heat flux profiles from the three tools but they all correspond very well to the measured heat flux data. Figure 5 shows the temperature fields computed with the three tools and reveals strong differences especially between CFX and the two other codes which may be attributed to the 
influence of the chemistry modeling. The CFX Flamelet solution shows in general lower temperatures than the equilibrium solution of Rocflam3 or the finite rate solution of TAU. Flame quenching processes which reduce the temperature are improbable at these locations behind the flame, because the turbulence intensity is rather small there. This difference in the thermal field is under investigation.

The combustion chamber pressure is a result of the present simulations, i. e., there is no pres-

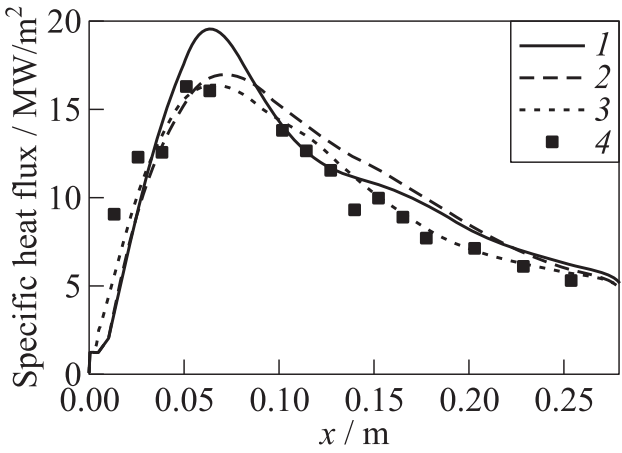

Figure 4 Wall heat flux distribution for the three applied tools $(1-$ TAU; $2-$ CFX Flamelet; and 3 - Rocflam3); 4 - test data sure boundary condition at the inlet or outlet. Numerical simulations prescribing the chamber pressure as inlet boundary conditions as often found in the literature are set up in an inconsistent manner. The simulations performed at Airbus

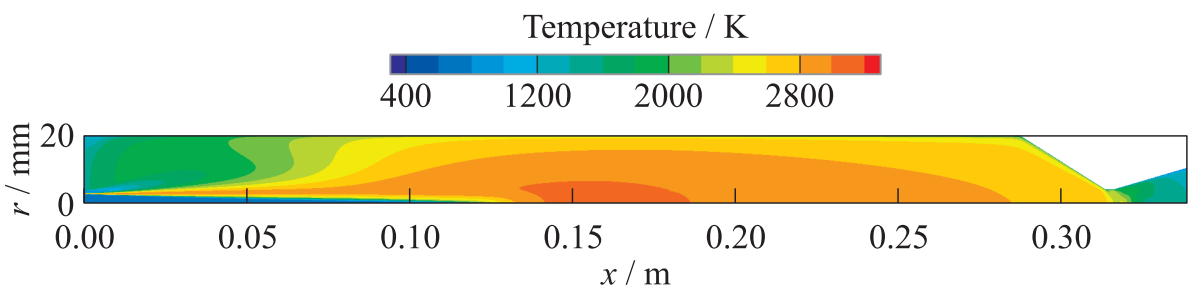

(a)

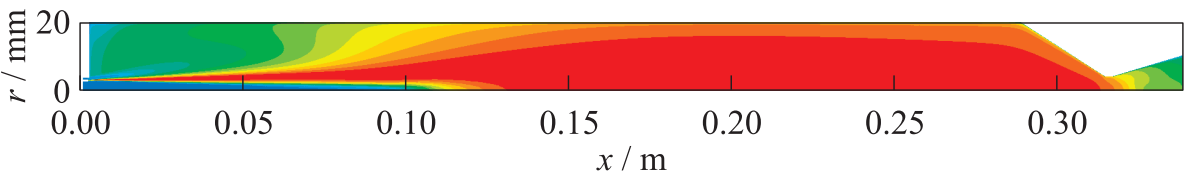

(b)

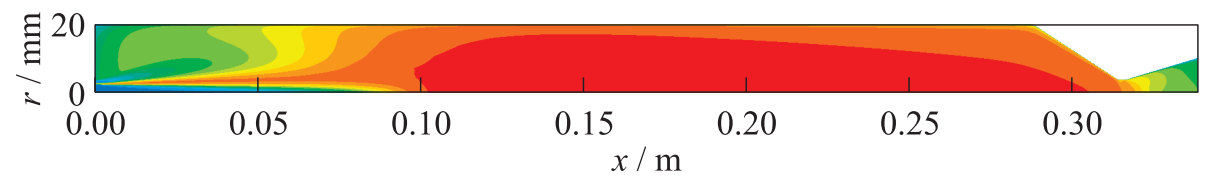

(c)

Figure 5 Temperature fields for the three applied tools: (a) CFX Flamelet; (b) TAU; and $(c)$ Rocflam3 
DS with CFX and Rocflam3 compute much lower combustion chamber pressures $\left(p_{c} \approx 5.0 \mathrm{MPa}\right)$ than the measured pressure $\left(p_{c} \approx 5.4 \mathrm{MPa}\right)$. TAU computes even a slightly lower combustion chamber pressure than the Airbus DS simulations of about 4.9 $\mathrm{MPa}$. Due to that, the combustion efficiencies of the simulations are much lower than $\eta_{c^{*}}$,Test computed from CEA2 and the experimental data. The simulations use the measured mass flow rates, inlet temperatures, and the species compositions from a complete combustion process in the preburner as boundary conditions. The composition of the complete preburner combustion is given in Table 1 and in [5] but as discussed in section 3 of this paper, the complete combustion in the preburners is questionable. However, all simulations performed so far are based on the assumption of complete combustion in both preburners. Considering incomplete combustion would result in a greater heat release inside the combustion chamber and would, thereby, increase the chamber pressure.

\section{PARAMETER STUDIES CONDUCTED DURING THE DEVELOPMENT OF THE TOOL SETTINGS}

In order to develop the settings for the simulations presented in the previous section, a number of parameter studies have been conducted with each of the applied tools. This section provides an insight into some of these studies.

Due to the fact that the Penn State combustor is a single element configuration and the characteristic length is very high, the outer recirculation zones (see e.g., Figs. 9 and 14) are much more intensive than in ordinary multiinjector configurations which usually possess much smaller distances between injector and chamber wall. The correct simulation of the very intensive recirculation zones, i. e., also the back-transport of the hot gases towards the face plate, is an essential feature which must be captured very accurately by the CFD tool in order to reproduce the measured data like the wall heat flux correctly. This section shows some of the parameter and sensitivity analyses on turbulent Prandtl and Schmidt numbers (Rocflam3), on grid convergence, turbulence and combustion modeling (TAU), and, finally, on the influence of the detailed numerical resolution of the inner injector flow (CFX).

\subsection{Investigation of the Influence of Different Turbulent Prandtl and Schmidt Numbers in Rocflam3}

The turbulent Prandtl and Schmidt numbers are inherent in RANS modeling (also, in unsteady RANS (URANS) and large-eddy simulation (LES)) and govern turbulent mixing and heat transfer. Thereby, the turbulent Prandtl number controls the wall heat flux process via the turbulent thermal conductivity and the 
turbulent Schmidt number controls the mixing process via turbulent diffusion. Since there are no definite figures for both quantities, they have to be stipulated by each model package individually. Therefore, it is appropriate to check their sensitivity range first. In Rocflam3, both parameters can be adjusted independently from each other. Figure 6 shows the wall heat flux profiles of Rocflam3 results for various turbulent Prandtl numbers.

As it is well known, the choice of the turbulent Prandtl number has a strong influence on the wall heat flux. By reducing the turbulent Prandtl number, the wall

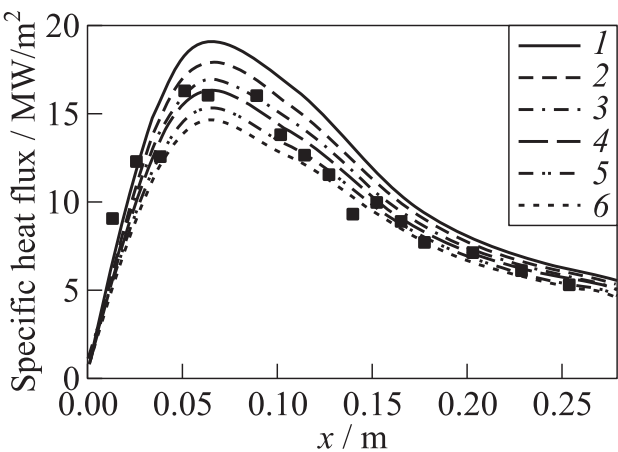

Figure 6 Wall heat flux distribution computed with Rocflam3; influence of a variation of the turbulent Prandtl number $\left(\mathrm{Sc}_{t}=0.6\right)$ : $1-\operatorname{Pr}_{t}=0.6 ; 2-0.7 ; 3-0.8 ; 4-0.9$; $5-1.0$; and $6-\operatorname{Pr}_{t}=1.1$. Signs refer to experiment heat flux increases mainly because of the increase of the effective thermal conductivity. In the Rocflam3 results shown in Fig. 6, the turbulent Schmidt number is always kept constant at $\mathrm{Sc}_{t}=0.6$. The shape of the heat flux distribution does not change in principle by varying the turbulent Prandtl number. This means that the axial position of the maximum wall heat flux is constant for all turbulent Prandtl numbers while there is only a shift to higher wall heat flux by reducing the turbulent Prandtl number. No influence on the aerodynamic field (streamlines) is visible when changing the turbulent Prandtl number, but the temperature field is slightly changing as it is shown in Fig. 7.

A higher turbulent Prandtl number leads to a broader and slightly more intensive high temperature region in the cylindrical part of the chamber, especially close to the walls. This is due to the fact that more heat is transferred from the fluid into the chamber wall.

Apart from the pure influence of the turbulent Prandtl number, also, the turbulent Schmidt number is very important. A separate variation of the turbulent Schmidt number has, therefore, been performed with Rocflam3. Figure 8 displays the wall heat flux distributions for different turbulent Schmidt numbers. Thereby, the turbulent Prandtl number is kept constant at 0.9.

A strong dependency of the wall heat flux on the turbulent Schmidt number is visible, but different to the turbulent Prandtl number: the turbulent Schmidt number mainly affects the shape of the heat flux profile. This means that the axial position of the maximum wall heat flux moves downstream by increasing the turbulent Schmidt number. As a consequence of this, it is clear that the 


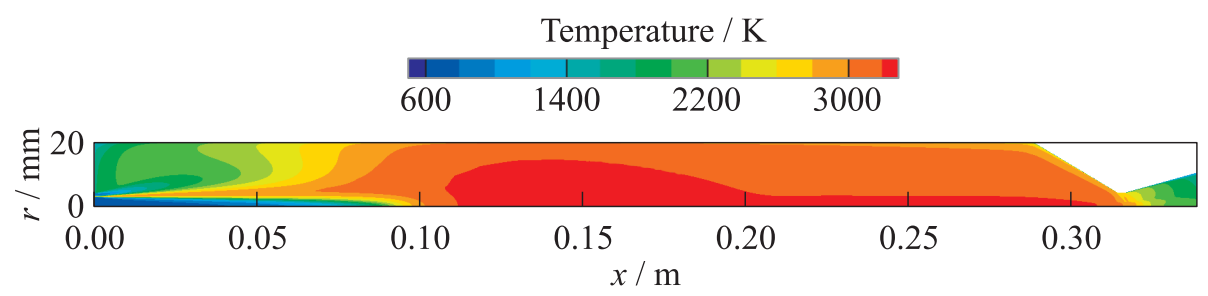

(a)

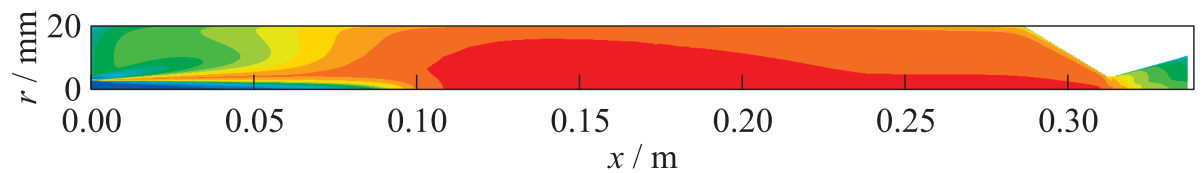

(b)

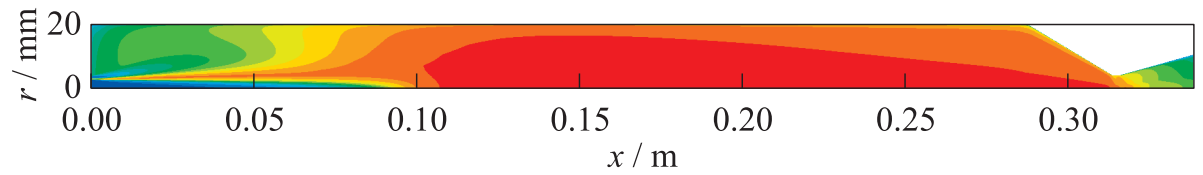

(c)

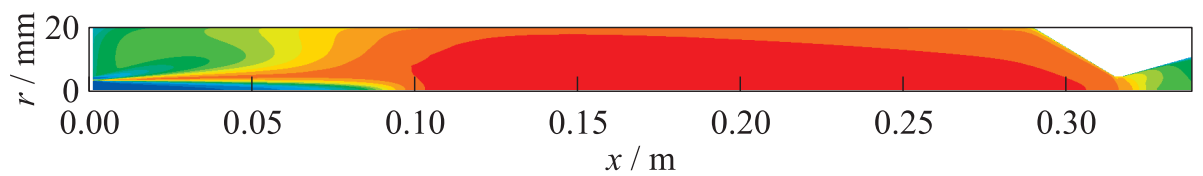

(d)

Figure 7 Temperature field computed with Rocflam3: influence of a variation of the turbulent Prandtl number $\left(\mathrm{Sc}_{t}=0.6\right):(a) \operatorname{Pr}_{t}=0.6 ;(b) 0.7 ;(c) 0.8$; and $(d) \operatorname{Pr}_{t}=0.9$

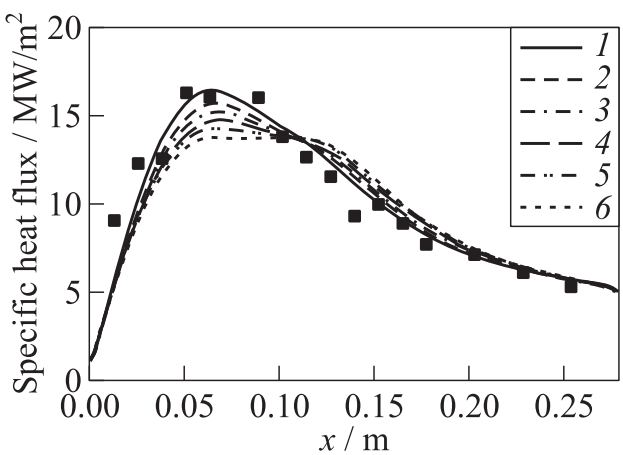

Figure 8 Wall heat flux distribution computed with Rocflam3; influence of a variation of the turbulent Schmidt number $\left(\operatorname{Pr}_{t}=0.9\right): 1-\mathrm{Sc}_{t}=0.6 ; 2-0.7 ; 3-0.8 ; 4-$ $0.9 ; 5-1.0$; and $6-\mathrm{Sc}_{t}=1.1$. Signs refer to experiment 


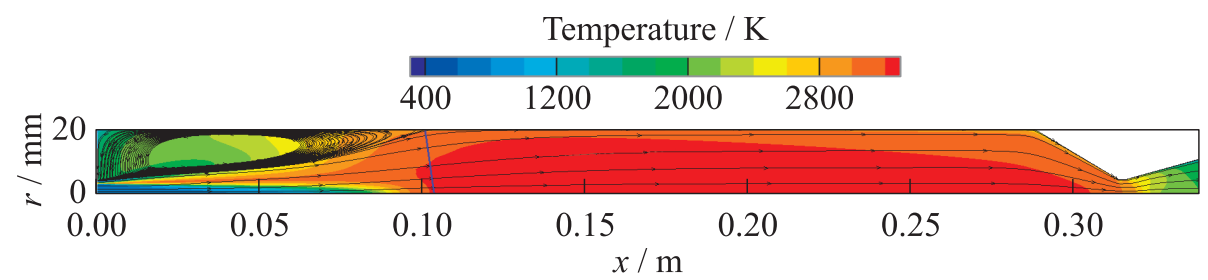

(a)

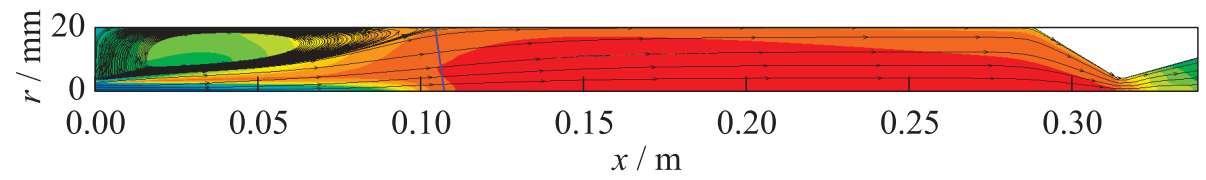

(b)

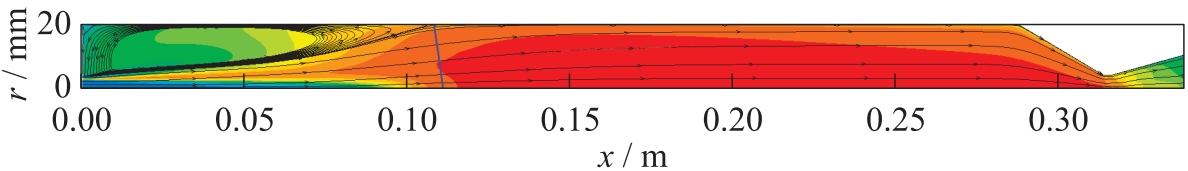

(c)

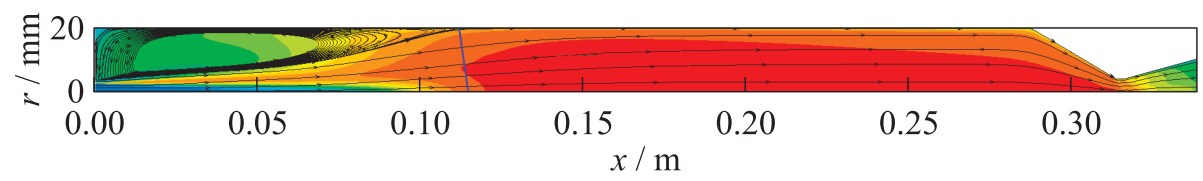

(d)

Figure 9 Temperature field and streamlines computed with Rocflam3; influence of a variation of the turbulent Schmidt number $\left(\operatorname{Pr}_{t}=0.9\right)$ : (a) Sc $\mathrm{c}_{t}=0.6 ;(b) 0.7 ;(c) 0.8$; and $(d) \mathrm{Sc}_{t}=0.9$

turbulent Schmidt number has not only an effect on the wall heat flux, it also influences the aerodynamic flow field, the mixing process, and due to this, also the combustion process. This effect is visualized in Fig. 9.

In Fig. 9, one can see that for higher turbulent Schmidt numbers, the outer recirculation zone expands. The hot gas stagnation point of the outer recirculation zone on the combustor wall is located further downstream for the higher turbulent Schmidt number (indicated by the blue line). By reducing the turbulent Schmidt number, the turbulent diffusivity is enhanced. This means that the mixing and combustion processes are also intensified. One can also see that the cold injection jet is a bit shorter for the lower turbulent Schmidt number $\left(\mathrm{Sc}_{t}=0.6\right)$ which is a direct consequence of the enhanced mixing by turbulent diffusivity. As a secondary effect of this, a faster combustion occurs for the lower turbulent Schmidt numbers. Such global influence is not observed for the turbu- 
lent Prandtl number where the influence is limited only to the energy transport which, however, is essential for the wall heat flux.

From these individual parameter variations, one could conclude that a tool package which assumes $\mathrm{Le}_{t}=1$, would predict the heat flux profile also fairly well with $\operatorname{Pr}_{t}=\mathrm{Sc}_{t}=0.85$ since the reduction of the turbulent Prandtl number to 0.85 could be compensated by an increase of the turbulent Schmidt number to 0.85 . At this place, it is recalled that the latter is the chosen setting for CFX.

\subsection{Grid Convergence and Variations of Turbulence and Combustion Modeling (TAU-Code)}

Different grid resolutions with a total number of points of 25,000 ("coarse"), 50,000 ("standard"), and 80,000 ("fine") were tested using the Spalart-Allmaras turbulence model in conjunction with the detailed chemistry combustion model (7-step reaction mechanism, neglect of turbulence-chemistry interaction). The grid topology is shown in Fig. 3 and described in section 4. The results in Fig. 10 show good agreement of the surface heat flux distributions for the different grid densities. The "standard" grid density was used for all further investigations.

The heat flux level of the Spalart-Allmaras results (used for the initial grid convergence study) in Fig. 10 is significantly too low and position of the heat flux peak is too far upstream. Due to these observed deficiencies, further parametric studies were carried out. Exemplary results from the application of a Menter SST turbulence model and different combustion models as described in section 4 are shown in Fig. 11. The main conclusions that can be drawn from the results

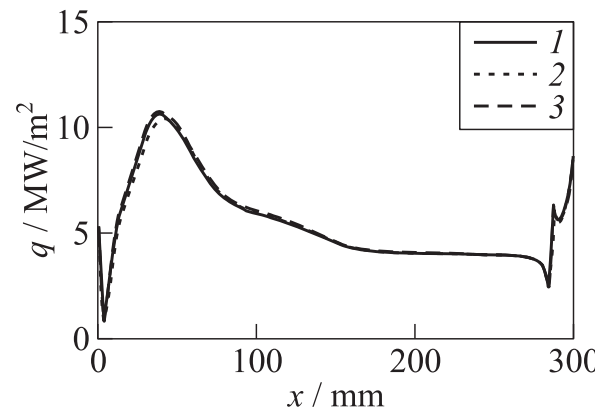

Figure 10 Wall heat flux distributions resulting from different grid densities: $1-$ standard grid; 2 - coarse grid; and 3 fine grid

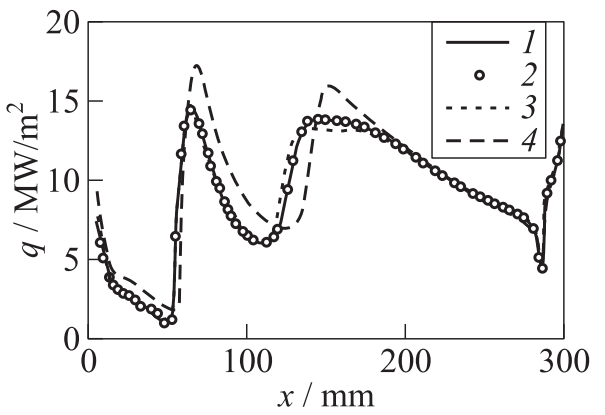

Figure 11 Application of different chemistry and combustion models: $1-7$-step chemistry; 2 - 19-step chemistry; 3 7-step chemistry + assumed PDF; and 4 - Flamelet model 
of Figs. 10 and 11 are that the turbulence model is of primary importance for the prediction of the wall heat flux and that the influence of the applied combustion model is comparatively small. The application of the Menter SST model without realizability limiter led to a qualitatively wrong prediction of the surface heat flux distribution including two distinct peaks. This is due to a large sensitivity of the structure of the recirculation zone in the upstream part of the combustor to the turbulence model. The difference between the 7-step and 19-step reaction mechanisms is negligible and the influence of the assumed-PDF modeling of the turbulence-chemistry interaction is small. This is consistent with previous numerical results for this test case [11]. The largest deviation is observed to occur between the detailed-chemistry and the Flamelet results. However, this deviation is small compared to the influence of the applied turbulence model.

All results obtained with the Spalart-Allmaras and Menter SST turbulence models do not reproduce the available experimental data for the surface heat flux distribution. Further investigations were carried out and a dramatic improvement of the computational result for the heat flux was observed by introducing the Durbin realizability constraint [13] to the Menter SST model. The resulting heat flux distribution is shown in Fig. 4 in section 5. The applied combustion model for this case was the 7-step laminar chemistry scheme. Both, the location of the heat flux peak and the total heat flux level, are well reproduced by this numerical model setup.

\subsection{Simulations with Resolved Injector in ANSYS CFX}

All CFX simulations shown up to now in this paper do not resolve the inner injection flow. In these simulations, the propellant inflow started at the injection plane (plane of the faceplate, see, e. g., Fig. 2) of the combustion chamber which is a simplification often done in CFD of combustion chambers. This results, of course, in block profiles for the velocity at the inlet. Resolving the inner injector flow, the velocity inlet profiles change towards parabolic profiles. Especially for certain parametric studies, it is usually too complex and time-consuming to resolve for every simulation the inner injector aerodynamics. Would the resolution, however, be necessary, it had to be transferred to multiinjector configurations too; otherwise, the conclusion drawn here is not conclusive for a design tool. But the influence of this inner injector flow has then to be investigated in order to justify correctly such simplifications. In this subsection, the inner injector flow is analyzed in detail and the influence on the processes and on the results inside the combustion chamber is evaluated in order to legitimate this simplification. Figure 12 shows the numerical mesh with the resolution of the injection element.

The numerical mesh which resolves the injection element is, again like the one without injection element, 2D (axially symmetric), and has in total 79,000 cells. Hereby, 16,000 cells (20\%) are used for the resolution of the inner flow of the 


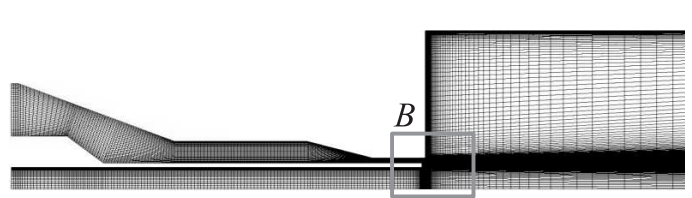

$A$

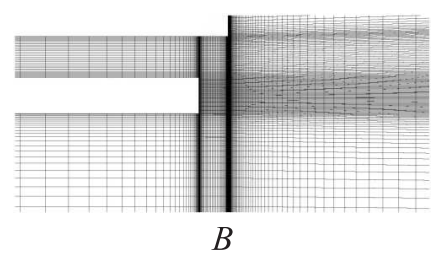

$B$

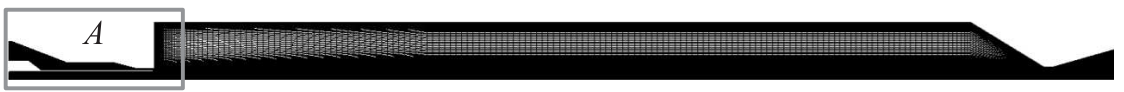

Figure 12 Numerical mesh with resolution of the injection element

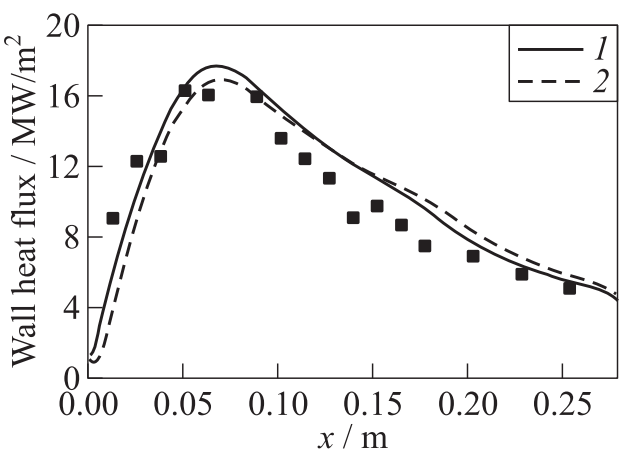

Figure 13 Wall heat flux; influence of the resolution of the injection element (chemistry model: CFX-Flamelet, turbulence model: CFX-SST): 1 - without injector; and 2 - with injector. Signs refer to experiment

injection element. As it can be seen in Fig. 12, the post-tip and the recess of the injection element are highly resolved with 52 cells in radial direction. This cell number is enough to resolve the vortex system which exists behind the post-tip and which is shown later in this paper. Figure 13 compares the wall heat flux distribution for simulations of CFX with and without the resolution of the inner injector flow.

The CFX simulation uses the Flamelet combustion model with a turbulent Prandtl number of $\operatorname{Pr}_{t}=0.8$, i. e., slightly lower than the number used for Fig. 4 result. In Fig. 13, one can see that there is a difference when resolving the inner injector flow compared to the solution without injector. But the difference is not very pronounced for the CFX solution. Especially further downstream, the results with and without resolution of injector are very similar. More important deviations are visible in the region where the wall heat flux has its maximum values, around the axial position of $X=0.07 \mathrm{~m}$. But here also the differences are not very pronounced. CFX shows here a deviation of around $7 \%$ in the 


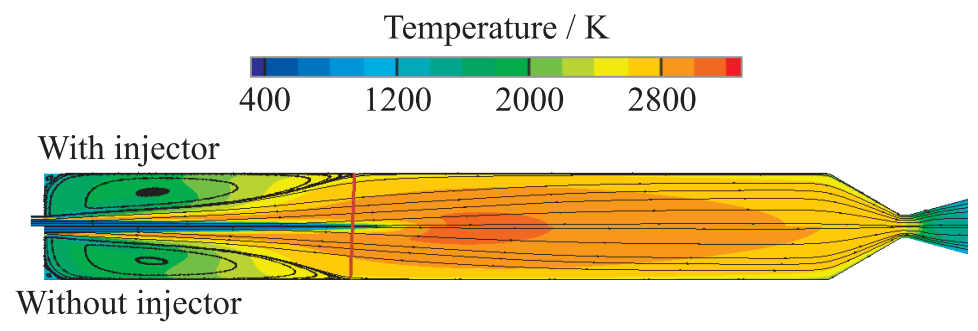

Figure 14 Temperature field (CFX-solution) with (upper frame) and without (bottom frame) resolution of injection element

maximum value when resolving the inner injector flow. CFX shows that in the first part of the combustion chamber, the wall heat flux is lower when resolving the inner injector flow. The highest difference is exactly at the position with the maximum wall heat flux which is close to the reattachment point. But further downstream, this picture changes. Here, the wall heat flux by resolving the inner injector flow is slightly higher. The comparison to the test data is, in general, very satisfying for all simulations. A more qualitative evaluation of the influence of the inner injector flow is shown in Fig. 14. Here, the temperature contour field is shown with overlaid flow streamlines. The top part of Fig. 14 shows the solution with resolving the inner injector flow and the bottom part without. The aerodynamics inside the combustion chamber are relatively similar, but one difference is obvious. The reattachment or stagnation point of the outer recirculation zone, indicated by the red line, is a bit further downstream in the case with resolution of the inner injector flow. As it was shown in Fig. 13, the maximum wall heat flux with resolution of the inner injector flow is slightly further downstream than without the inner injector flow. This is in-line with the results of Fig. 14 where the position of the stagnation point is shown.

Also, for the evaluation of the mixing process, the detailed flow field directly behind the post-tip is essential. The resolution of the inner injector flow has a contribution, but the dominant factor is the flow flied and the turbulence production in the region downstream of the post-tip. Figure 15 shows the aerodynamic field at the post-tip region with the flame anchoring at the tip for both CFX results.

The CFX solution shows very good and stable convergence for the case shown in Fig. 15. The vortex system directly behind the liquid oxygen ( $\mathrm{LOx}$ ) tip is resolved well and the turbulence production by this vortex system is essential for the mixing behavior and due to that also for the combustion process further downstream in the reactive shear layer. The combustion intensity in the shear layer determines the wall heat flux but obviously, the main features can be reproduced also not by resolving the details of the inner injector flow as long as the aerodynamics behind the LOx tip are simulated accurately. 


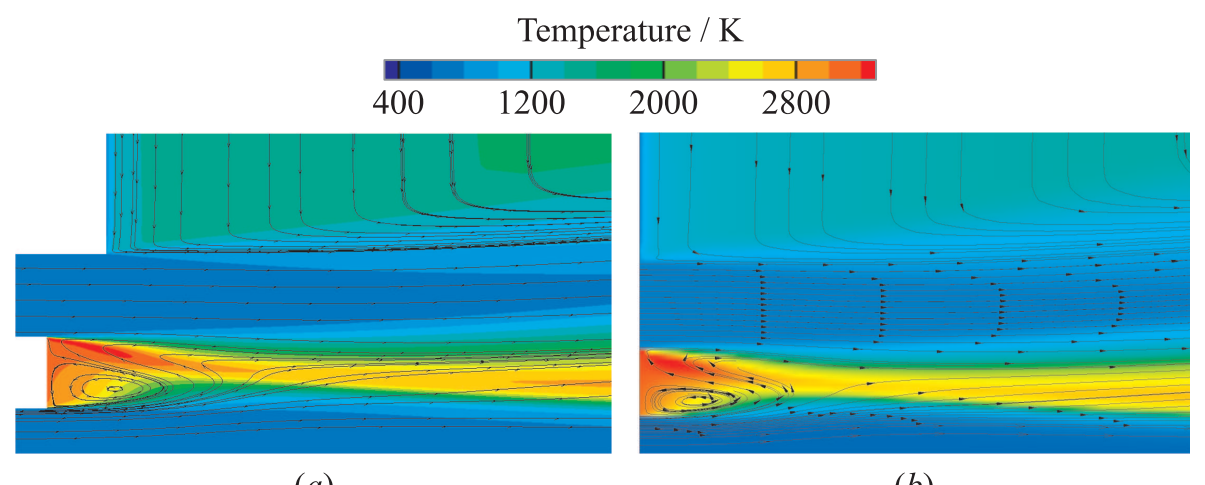

(a)

(b)

Figure 15 Vortex system behind the post-tip with $(a)$ and without $(b)$ resolution of inner injector flow. Combustion model: CFX-Flamelet; turbulence model: CFX-SST

\section{CONCLUDING REMARKS}

This paper presents the simulation results for the Penn State test case obtained with three different CFD tools: the Airbus DS in-house solver Rocflam3, the commercial solver CFX adapted for rocket applications by Airbus DS, and the DLR code TAU. All three tools are able to compute the wall heat flux profile achieving good agreement with the available test data.

The axial position of the heat flux peak is well captured. An overestimation of the wall heat flux simulated by TAU of about $20 \%$ occurs in the vicinity of the heat flux peak and of about $10 \%$ in the downstream section of the combustion chamber. A large influence of the applied turbulence model is observed. In particular, the application of the Durbin realizability limiter to the Menter SST model leads to a significantly improved prediction of the surface heat flux distribution.

From the conducted investigations, it can be concluded that the choice of the turbulence model has major impact on the solution just as the selection of the chemistry model and the choice of the turbulent transport characteristics $\mathrm{Pr}_{t}$ and $\mathrm{Sc}_{t}$ for which no specific numbers exist. This is not surprising since the huge outer recirculation zone triggered by the rocket thrust chamber untypically large contraction ratio of the combustor governs the flow field evolution. This is also a reason why this test case is very challenging. Converged solutions are obtained with CFX using the SST with and without resolution of the inner injector flow. The investigation discussed in subsection 6.3 reveals that it is not necessary to resolve the inner injector flow as long as the aerodynamics behind the LOx tip and the processes inside the reactive shear layer are resolved accurately. This means that a lot of computation time can be saved by neglecting the inner in- 
jector flow. The correct resolution of the vortex system behind the LOx tip is essential because this determines the turbulence production in the shear layer which is the dominant process driving the mixing and combustion in the reactive shear layer. The main outcome is that these dominant parameters in the reactive shear layer can also be captured without detailed resolution of the inner injector flow but adequate resolution of the LOx-post and the reactive shear layer.

Moreover, there are still some uncertainties which prohibit the full understanding of the test case. The test data show some inconsistencies, i. e., the measured mass flow rates do not fit to the measured combustion pressure. An incomplete combustion in the preburners could be one possible contribution to this mismatch. However, there may also be other reasons that cannot be quantified.

Finally, the results shown in this paper clearly justify the application of RANS models for this test case. This means that it is not necessarily essential to apply more sophisticated model approaches like URANS or LES, as it is stated in other publications, e.g., in [3] or [4]. This is crucial for all tool developers designing a tool for multiinjector combustor layout and optimization work.

\section{ACKNOWLEDGMENTS}

This work was performed within the National Technology Program TARES. This program is sponsored by the German Space Agency, DLR Bonn, under contract No. 50RL1210.

\section{REFERENCES}

1. Marshall, W.M., S. Pal, R.D. Woodward, and R. J. Santoro. 2005. Benchmark wall heat flux data for a $\mathrm{GO}_{2} / \mathrm{GH}_{2}$ single element combustor. 41st AIAA/ASME/SAE/ASEE Joint Propulsion Conference \& Exhibit. Tucson. AZ, USA.

2. Pal, S., W. Marshall, R. Woodward, and R. Santoro. 2006. Test case RCM 1: Penn State preburner combustion - wall heat flux data for a $\mathrm{GO}_{2} / \mathrm{GH}_{2}$ uni-element combustor. 3rd Workshop (International) Rocket Combustion Modeling. Paris.

3. Lian, C., and C. L. Merkle. 2010. Contrast between steady and time-averaged unsteady combustion simulations. 48th AIAA Aerospace Sciences Meeting Including the New Horizons Forum and Aerospace Exposition. Orlando, FL, USA.

4. Lempke, M., P. Gerlinger, M. Aigner, and M. Rachner. 2011. Steady and unsteady RANS simulations of cryogenic rocket combustors. 49th AIAA Aerospace Sciences Meeting. Orlando, FL, USA.

5. McBride, B. J., and S. Gordon. 1996. Computer program for calculation of complex chemical equilibrium compositions and applications. Cleveland, OH, USA. NASA Reference Publication 1311.

6. Frey, M., T. Aichner, B. Ivancic, B. Kniesner, and O. Knab. 2010. Modeling of rocket combustion devices. 10th AIAA/ASME Joint Thermophysics and Heat Transfer Conference. Chicago, IL, USA. 
7. Ivancic, B., H. Riedmann, and M. Frey. 2012. Validation of turbulent combustion models for $3 \mathrm{D}$-simulations of liquid $\mathrm{H}_{2} / \mathrm{O}_{2}$ rocket combustors. Space Propulsion Conference. Bordeaux, France.

8. Menter, F. R. 1994. Two-equation eddy-viscosity turbulence models for engineering applications. AIAA J. 32(8):1598-1605.

9. Menter, F. R. 2009. Review of the shear-stress transport turbulence model experience from an industrial perspective. Int. J. Comput. Fluid Dyn. 23(4):305-316.

10. Brokaw, R. 1964. Approximate formulas for viscosity and thermal conductivity of gas mixtures. Washington, DC, USA: NASA. 17 p.

11. Lempke, M., P. Gerlinger, and M. Aigner. 2013. Assumed PDF modeling of rocket combustor simulations. Progress in propulsion physics. Eds. L. DeLuca, C. Bonnal, O. Haidn, and S. Frolov. Eucass advances in aerospace sciences ser. Moscow-Paris: EDP Sciences - TORUS PRESS. 4:569-582.

12. Launder, B. E., and B. I. Sharma. 1974. Application of the energy-dissipation model of turbulence to the calculation of flow near a spinning disc. Lett. Heat Mass Trans. 1:131-138.

13. Durbin, P. 1996. On the $k$-epsilon stagnation point anomaly. Int. J. Heat Fluid Flow 17:89-90.

14. Yap, C. 1987. Turbulent heat and momentum transfer in recirculating and impinging flows. Manchester, U.K.: Faculty of Technology, University of Manchester. PhD Thesis.

15. German Aerospace Center, DLR. 2014. Homepage of DLR TAU Code. Available at: http://tau.dlr.de (accessed June 8, 2015).

16. Spalart, P.R., and S. Allmaras. 1992. A one-equation turbulence model for aerodynamic flows. AIAA Paper No. 92-0439.

17. Hannemann, V. 1997. Numerische Simulation von Stoß-StoßWechselwirkungen unter Berücksichtigung von chemischen und thermischen Nichtgleichgewichtseffekten. German Aerospace Center, DLR. DLR-Forschungsbericht 97-07.

18. Gerlinger, P. 2001. An implicit multigrid method for turbulent combustion. J. Comput. Phys. 167:247-276.

19. Gaffney, R., J. White, S. Girimaji, and J. Drummond. 1992. Modeling turbulence chemistry interactions using assumed PDF methods. 28th AIAA/SAE/ASME/ASEE Joint Propulsion Conference and Exhibit.

20. Karl, S. 2011. Numerical investigation of a generic scramjet configuration. Technical University Dresden. PhD Thesis. Available at: http://nbn-resolving.de/ urn:nbn:de:bsz:14-qucosa-68695 (accessed June 8, 2015).

21. Laurence, S., S. Karl, J. Martinez Schramm, and K. Hannemann. 2013. Transient fluid-combustion phenomena in a model scramjet. J. Fluid Mech. 722:85-120.

22. Oevermann, M. 1998. Ein Finite-Volumen Verfahren auf unstrukturierten Dreiecksgittern zur Berechnung turbulenter Diffusionsflammen in kompressiblen Strömungsfeldern. German Aerospace Center, DLR. DLR-Forschungsbericht 98-01.

23. Pitsch, H. FlameMaster v3.3.10: $\mathrm{A} \mathrm{C}++$ computer program for $0 \mathrm{D}$ combustion and 1D laminar flame calculations. Available at: http://www.stanford.edu/group/ pitsch/FlameMaster.htm. (accessed June 8, 2015).

24. Pitsch, H. 1993. Entwicklung eines Programmpaketes zur Berechnung eindimensionaler Flammen am Beispiel einer Gegenstromdiffusionsflamme. Lehrstuhl für Technische Mechanik, RWTH Aachen. Diplomarbeit. 